\title{
Drug nanotargeting for treatment of neurodegeneration and aging
}

\author{
Joseph S. D'Arrigo ${ }^{\mathrm{a}, *}$ \\ ${ }^{a}$ Cavitation-Control Technology Inc., Farmington, CT 06032, USA.
}

\begin{abstract}
Microvascular endothelial dysfunction precedes, often by decades, the cognitive decline associated with Alzheimer's disease. Accordingly, cerebrovascular risk factors (e.g., atherosclerosis, diabetes, aging) contribute to the pathogenesis of this common neurodegenerative disease. By incorporating appropriate drug(s) into biobased (lipid cubic phase) nanocarriers, one obtains a multitasking combination therapeutic which targets certain cell-surface scavenger receptors, mainly class B type I (i.e., SR-BI), and crosses the blood-brain barrier. This (intravenous) combination therapeutic, known to be a successful drug carrier, would make it possible for various cell types within the brain (all potentially implicated [see below] in Alzheimer's disease) to be simultaneously nanotargeted for localized drug delivery via cell-surface SR-BI. Hence, such colloidal-nanocarrier targeting allows for various Alzheimer's-related cell types to be simultaneously searched in a holistic integrative approach. This (colloidal-nanocarrier) targeting advantage, in vivo, may be particularly important when delivering pleiotropic natural substances (e.g., a flavonoid) or for repurposing an FDA-approved drug. For example, the described/proposed colloidal (nanoemulsion) nanocarrier is especially useful for the delivery of low-molecular-weight compounds having a significant degree of lipophilicity (i.e., low water solubility), such as various pleiotropic natural substances (e.g., the plant polyphenol known as "resveratrol", commonly used as a dietary supplement).
\end{abstract}

Keywords: Alzheimer's disease, cognitive impairment, nanoemulsion, SR-BI, vascular dementia

\section{Introduction}

Vascular brain lesions occur frequently in people over 70 years old, and recent reviews [1,2] provide much evidence that a large percentage of dementia cases may be associated with cerebrovascular disease $[3,4]$. Accordingly, vascular cognitive impairment is the second leading cause of dementia behind Alzheimer's disease, and often is a comorbidity in the Alzheimer's patient $[5,6]$. Furthermore, growing data from various animal models indicate that cerebrovascular dysfunction often precedes cognitive impairment as well as the onset of neurodegenerative changes in Alzheimer's disease [2,4].

In pathological states, members of the scavenger receptor family of proteins (including class A receptors and class B

* Corresponding author: Joseph S. D'Arrigo

Mailing address: Cavitation-Control Technology Inc., Bellevue, WA 98007, USA.

Email: cavcon@ntplx.net

Received: 22 February 2021 / Accepted: 14 April 2021 receptors) mediate the recruitment, activation and transformation of macrophages, and other cells which appear to be related to the development of not only Alzheimer's disease but also atherosclerosis [2]. Lipid accumulation in the blood vessel wall depends on the intracellular uptake by macrophages, which transform into foam cells. Overloaded foam cells finally degenerate, leaving extracellular lipid deposits. The lipid overload of macrophages is brought about by several classes of cell-surface scavenger receptors. As one example, a major type of class B scavenger receptor is reported to be upregulated; hence, binding followed by uptake perpetuates a cycle of lipid accumulation and receptor expression. Both class B and class A scavenger receptors are expressed in the lipid-laden macrophages in atherosclerotic lesions. Furthermore, the differential distribution of the scavenger receptor types within human atherosclerotic lesions has already been reported in the literature [2]. In view of the detailed published information available on the presence, functional characteristics and localization of scavenger receptor populations in atherosclerotic lesions, localized drug delivery to such lesions may offer a means for targeted drugdelivery therapy of atherosclerosis and, potentially also (cf. above), late-onset Alzheimer's disease. 
Endothelial dysfunction and targeted nanotherapy for late-onset dementia

Small-vessel disease is commonly found in patients who have other brain pathologies, such as plaques and tau tangles associated with neurodegenerative disease. Accordingly, the most common cause of clinical dementia in the elderly has been ascribed to mixed pathology, displaying both Alzheimer's disease and vascular abnormalities. Bennett et al. [5] have recently pointed to much evidence that, as observed in the clinic by MRI scans or at autopsy by neuropathological evaluation, "pure" Alzheimer's disease is substantially less common than mixed dementias -- where protein tau tangles (in neurons) and (extracellular amyloid-beta) plaques are accompanied by vascular changes. These authors' own published work provides evidence that tau pathological changes (in neurons) can impact brain endothelial-cell biology which, in turn, induces changes in the brain's microvasculature (including abnormal spiraling morphologies, reduced blood vessel diameters, and increased overall blood vessel density in the cerebral cortex) separate from the effects of senile plaques on vasculature; hence, these observations indicate a previously unknown pathway by which pathological tau tangles may accelerate cognitive decline in Alzheimer's disease [5]. In Alzheimer's disease itself, the characteristic lesions that develop, called senile plaques, are extracellular deposits principally composed of insoluble aggregates of amyloid- $\beta$ protein $(A \beta)$ fibrils, infiltrated by reactive microglia and astrocytes. A $\beta$ fibrils exert a cytotoxic effect on neurons and stimulate microglia to produce neurotoxins, such as reactive oxygen species. Mononuclear phagocytes, including microglia, express scavenger receptors that mediate adhesion and/or endocytosis; in particular, microglia have been shown to be intimately associated with amyloid deposits, and have also been implicated as scavengers responsible for clearing $A \beta$ fibril deposits of Alzheimer's disease. Accordingly, microglial scavenger receptors have already been described as novel targets for therapeutic interventions in Alzheimer's disease; specifically, it is believed that microglia play a major role in the cellular response associated with the pathological lesions of Alzheimer's disease and, furthermore, that pharmacological agents which suppress microglial activation may prove a useful strategy to slow the progression of Alzheimer's disease [2]. The vascular changes associated with small-vessel disease of the cerebral microvasculature include morphological alterations and resultant bloodbrain barrier (BBB) breakdown. It is not surprising, therefore, that multiple epidemiological studies have revealed a marked overlap among risk factors for small-vessel cerebrovascular disease and late-onset Alzheimer's disease [2]. It has been reported continually that endothelial modulation and/or repair is practicable by pharmacological targeting [1,2,7-13] via cell-surface scavenger receptors (mainly class B type I, i.e., SR-BI) [13-15]. Since SR-BI has already been identified as a major receptor for highdensity lipoprotein or HDL (with their major apolipopro- tein (apo)A-I), as well as for the recently reviewed $[1,2]$ "lipid-coated microbubble/nanoparticle-derived" (LCM/ ND) nanoemulsion (see below), this multitasking lipid nanoemulsion can arguably serve as a targeted, apoA-Ibased, (SR-BI mediated) therapeutic agent for common (late-onset) dementias [16-18]. Documented similarities in the lipid composition of HDL versus these nanoemulsions (drug-carrier) particles may enable such LCM/ND nanoemulsions to mimic, in part, HDL-particle heterogeneity $[1,2]$.

This targeted-delivery-approach, using the proposed LCM/ND lipid nanoemulsion for treating the more common (late-onset) dementias, receive added impetus from continual findings of cerebrovascular pathology [1,19-29] and an apparent endothelium dysfunction [2,17,18,25,30$36]$ in both Alzheimer's disease and its major risk factors [1,2,29-41]. Incorporating certain drug molecules into the LCM/ND lipid nanoemulsion type (yielding particle sizes mostly $<0.1 \mu \mathrm{m}$ in diameter - see Figure 1), known to be a successful drug carrier $[2,42,43]$, would make the following possible: Various cell types, all potentially implicated in Alzheimer's disease [1], can be simultaneously nanotargeted via cell-surface SR-BI $[42,43]$.

\section{LCM/ND lipid nanoemulsion: Particle size dis- tribution and safety studies}

Physical characterization of the actual size distribution, of the LCM/ND lipid nanoemulsion particles, has been described in detail earlier [2]. Basically, collaborative multidisciplinary analyses of the nanoemulsion particle sizes, particularly in the submicron range, were carried out using three different techniques, i.e., optical particle counting (see below), dynamic light scattering, and scanning electron microscopy; the first of these techniques yielded the most data. Five different optical particle counters, all manufactured by Particle Measuring Systems (Boulder, $\mathrm{CO}$ ), were used to measure the scattered light over different collection angles. The filtered LCM/ ND lipid nanoemulsion contained close to $10^{10}$ particles $<0.10 \mu \mathrm{m}$ per $\mathrm{ml}$, with most $(\sim 90 \%)$ of the nanoemulsion particles being smaller than $0.2 \mu \mathrm{m}$ in diameter (Figure 1) [2]. The effect of concentration on the nanoemulsion size distributions was also determined using the S100 optical particle counter. It was thought that if the total concentration of LCM/ND nanoemulsion material changed, the size distribution might also change as a new equilibrium condition was achieved. All of the measurements were found to be essentially identical, indicating that the LCM/ ND lipid nanoemulsion did not change particle size when subjected to the different concentration conditions. In addition, the effect of LCM/ND nanoemulsion age on the particle size distribution was determined by measuring the nanoemulsion size distribution at different times over 37 days, using the M65 optical particle counter. No change in the size distribution with time (over at least 1 month) was evident [2]. In summary, the LCM/ND nanoemulsion size 


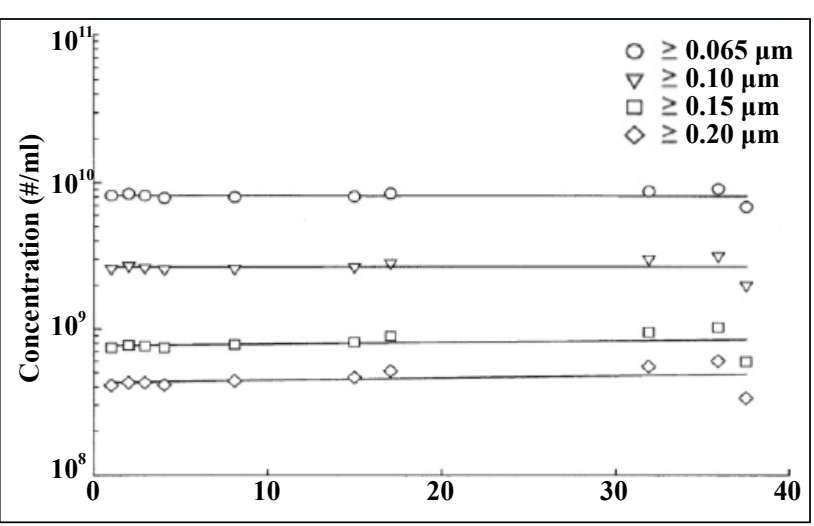

Figure 1. LCM/ND nanoemulsion stability over time. (Adapted from ref. 2)

distribution in deionized water was measured using several analytical techniques. This nanoemulsion was found to contain close to $10^{10}$ particles $<0.1 \mu \mathrm{m}$ per $\mathrm{ml}$ when measured using optical particle counters. A large majority $(\sim 90 \%)$ of the nanoemulsion particles were smaller than $0.2 \mu \mathrm{m}$ in diameter.

With regard to safety considerations, the LCM/ND lipid nanoemulsion particles have neither been found to agglomerate nor coalesce into any "superparticle or microbubble-like" structure larger than $5 \mu \mathrm{m}$, either in vitro or in vivo, thus the risk of embolus is negligible [2]. In addition, acute intravenous toxicity studies of this (isotonic) LCM/ND nanoemulsion agent in rabbits and dogs were conducted at an independent GLP contractor. [Note that the acronym "GLP" indicates that studies conducted by the aforesaid contractor are in compliance with the Good Laboratory Practices Regulations as stated in 21 CFR Part 58, for submission to the U.S. Food and Drug Administration in support of an Investigational New Drug Application (INDA). Accordingly, this statement carries the expectation that there are no deviations from the GLP regulations which would affect the quality or integrity of the study.] The acute intravenous LD50 in both species was found to be greater than $4.8 \mathrm{ml} / \mathrm{kg}$. Furthermore, no signs of gross toxicity or mortality were observed at a dosage of $4.8 \mathrm{ml} / \mathrm{kg}$. It has also been found in other animals (rangefinding subchronic intravenous) toxicology studies, using this same (isotonic) lipid nanoemulsion agent, that at intravenous doses of $0.14 \mathrm{ml} / \mathrm{kg}$ given three times per week for 6 weeks in rats and, separately, at intravenous doses of $0.48 \mathrm{ml} / \mathrm{kg}$ given three times per week for 3 months in rabbits, the following toxicology results were observed: There were no untoward changes in serum chemistry, liver functions, hematology, or clotting profile or histological changes in adrenals, bladder, brain, heart, kidney, liver, lungs, marrow, pituitary, spleen, testes, thyroid, or ureters [2]. Finally, the lipids present in the LCM/ND nanoemulsion agent are similar to those found in the clinical products Intralipid and Liposyn III, except at extremely smaller concentrations and dosages employed than with these two human-approved clinical products. Intralipid is a fat emulsion for intravenous administration; in particular, the saturated fatty acids and neutral triglycerides and/ or di- and monoglycerides present are metabolized by the same metabolic pathways. Hence, a comparison of the lipid composition of LCM/ND nanoemulsion agent with that of the intravenous fat-emulsion products (Intralipid and Liposyn), and their respective (intended clinical) dosages, indicate enormous margins of safety for the LCM/ ND nanoemulsion in its intended clinical application for targeted drug-delivery therapy in humans [2].

\section{LCM/ND nanoemulsion type and targeting via lipid cubic phases}

Monoglyceride is the largest fraction (by wt. \%) of the lipids used to produce (Filmix ${ }^{\circledR}$ ) LCM/ND nanoemulsions. As a group, monoglycerides exhibit the ability to selfassemble into varied and useful dispersed cubic phases (among other liquid-crystalline phases) when placed in contact with water $[2,44,45]$. The (lyotropic or solventinduced) cubic liquid-crystalline phases can be grouped into two distinct classes: bicontinuous cubic phases [46] and micellar or discontinuous (e.g., type $\mathrm{Fd} 3 \mathrm{~m}$ ) cubic phases [47]. A noteworthy lipid cubic phase of the latter category is based upon packings of discrete inverse micellar aggregates and is formed by a variety of lipid systems [47]. Seddon et al. [48] point out that the most frequently observed such (inverse micellar cubic) structure is a cubic phase of crystallographic space group $\mathrm{Fd} 3 \mathrm{~m}$, which requires a heterogeneous mixture of polar lipids [49,50]. The dispersed $\mathrm{Fd} 3 \mathrm{~m}$ cubic phase is particularly relevant to the earlier-described LCM/ND lipid nanoemulsion; specifically, both (above-described) lipid structures often specifically include cholesterol and three categories of (saturated) glycerides, that is, tri-, di-, and monoglycerides $[51,52]$.

In this particular targeted-delivery approach, the self-assembled "lipid particle" structure itself (upon intravenous injection of the LCM/ND nanoemulsion) is apparently successfully utilized as the "active" targeting ligand which is directed via (adsorption of) plasma lipoproteins, including notably apoA-I, toward the appropriate receptors on the target-cell surface. This likely adsorption of apoA-I and, hence, targeting success of these nanoemulsion particles is understandable [2]. When the above information is combined with the known heterogeneity of HDL particles as well as the well-documented multiligand capability of SR-BI, then again SR-BI emerges as the prime candidate (of all lipoprotein receptors) for major involvement in the enhanced endocytosis of LCM/ND nanoemulsion particles into, and transcytosis across, the endothelial-cell layer of the BBB [2].

\section{Brain Injury, Edaravone, Resveratrol, and Alzheimer's Disease}

Besides considerations about amyloid pore formation (regarding calcium fluxes and Alzheimer's disease) described 
in the literature (e.g., [53-58]), and especially important pathophysiological overlap exists between traumatic brain injury (TBI) and Alzheimer's-disease brain. Interestingly, the drug Edaravone has been used successfully, in past TBI research, due to its neuroprotective and antioxidative effects on the brain after TBI. These pharmacological effects lead to a decreased inflammatory response and decreased glial activation, thereby reducing neuronal death and improving neurological function [59,60]. [Edaravone continues to be used clinically to aid patient recovery from ischemic stroke in Japan since 2001, and this drug was also approved for the treatment of amyotrophic lateral sclerosis (ALS) in the USA. The drug is thought to reduce oxidative stress in cells by lowering intracellular levels of free radicals; it is administered by intravenous infusion and is primarily excreted in the urine. Edaravone is available commercially as a clear, colorless liquid provided as a sterile injection solution. The drug is metabolized to pharmacologically inactive sulfate and glucuronide conjugates. The pharmacokinetics of Edaravone was not affected by age in the geriatric population during clinical trials, and no significant differences were observed between Japanese and Caucasian subjects (cf. [59,60]).]

In view of the above description of TBI, the effects of the drug Edaravone, and the pathophysiological overlap of TBI with many characteristics of Alzheimer's disease brain (cf. above), it is logical and consistent that Jiao et al. [61] have recently reported that Edaravone can also ameliorate Alzheimer's disease-type pathologies and cognitive deficits of a mouse model of Alzheimer's disease. These investigators further state that their above findings suggest that Edaravone is a promising drug candidate for Alzheimer's disease by targeting multiple key pathways of the disease pathogenesis [61] (cf. [62]).

While the risk factors for dementia trigger widespread inflammation and oxidative stress (e.g., $[63,64]$ ), it is also true that these two processes can result in more biological effects than enhanced calcium load in brain tissue and neurodegeneration (cf. [65-67]). In fact, oxidative stress and inflammation each involve pathophysiological cascades associated with a wide range of pathologies and especially aging [67]. Accordingly, Khalil et al. [68] found that Alzheimer's disease impaired the interaction of HDL (and apoA-I) with the SR-BI receptor, and their experimental results indicated that such patients had higher levels of oxidative stress $[68,69]$.

Lastly, in addition to the above-described repurposing of the FDA-approved drug Edaravone (cf. Abstract and this Section, para. 1\&2), it was alluded to earlier that targeted delivery of pleiotropic natural substances (cf. Abstract), such as docosahexaenoic acid (DHA) [42], astaxanthin [70], and resveratrol (e.g., [71-73]) - fit well with the initial drug candidates (for incorporation into the LCM/ ND lipid nanoemulsion) already suggested elsewhere [1]. These suggested drug candidates, chosen on the basis of their low-molecular weight and sufficient lipophilicity (as again proposed here), would be intended for use in the targeted treatment of Alzheimer's disease and late-onset de- mentia. As particularly concerns resveratrol, Broderick et al. [71] have recently emphasized that this plant polyphenol has gained interest as a nonpharmacological (dietary) approach for the treatment and prevention of Alzheimer's disease. Resveratrol is a major ingredient found in red wine, grape seeds, and certain nuts and berries; (e.g., [71]) this plant compound is a type of natural phenol [i.e., a flavonoid] commonly used as a dietary supplement. When surveying the dietary literature, available evidence from cell culture experiments indirectly implies that many of the effects of flavonoids, such as anti-inflammatory and/ or neuroprotective activities, are related to their ability to modulate cell-signaling pathways. In fact, intracellular concentrations of flavonoids required to influence cellular signaling are observed to be considerably lower than those required to influence cellular antioxidant capacity. Hence, flavonoid metabolites may retain their ability to interact with cell-signaling proteins even if their antioxidant activity is reduced (cf. [71-73]). Broderick et al. cite published evidence that resveratrol attenuates learning impairment and delays the onset of neurodegeneration in transgenic murine models of Alzheimer's disease; moreover, a significant reduction in the number of activated microglia and decreased inflammation in APP/PS1 mice following resveratrol treatment has been reported [71]. In summary, the above-described targeting advantage, using the LCM/ND lipid nanoemulsion, may be particularly important when delivering pleiotropic natural substances or for repurposing an FDA-approved drug in order to now treat late-onset dementia.

\section{Conclusions}

A desirable feature for a parenteral lipid nanoemulsion is the ability to avoid "reticuloendothelial system" (RES) capture and hence circulate for a prolonged period in the bloodstream, thereby allowing the opportunity for cellselective targeting of drugs. Accordingly, it is interesting to consider much evidence in the literature, reviewed elsewhere [2], which indicates that a sizable portion of intravenously injected LCM/ND lipid nanoemulsion bypasses the RES to then become endocytosed by target cells. One probable reason for this repeated experimental observation is that the colloidal nanoparticles employed are uncharged (in such lipid nanoemulsions) and, hence, these dispersed liquid-crystalline phases are all unlikely to be captured easily by the RES during circulation (prior to adsorption of plasma apoA-I and subsequent receptormediated endocytosis) [2]. More specifically, in vivo biodistribution studies (using micellar nanoparticles) have demonstrated that undesirable liver uptake was very large for highly positively or negatively charged (bloodborne) nanoparticles; based on these studies, the authors conclude that very low charge at the nanoparticle surface reduces the undesirable clearance by the reticuloendothelial system (RES) and improves the blood compatibility [74]. This conclusion is consistent with earlier published 
work, by other investigators, indicating that charge neutrality at the surface of blood-borne nanoparticles greatly increases their blood-tissue compatibility [75]. Another factor that can prolong the half-life in the bloodstream, of a circulating lipid nanoemulsion, relates to particle size; namely, the size of the lipid emulsion particles themselves is known to influence the RES uptake of intravenous lipid emulsions. Various investigators have reported that "small-particle" ( $\sim 0.10 \mu \mathrm{m}$ in diameter) lipid emulsions displayed: (1) a reduced hepatic uptake; and (2) a much slower plasma clearance, or a significantly greater area under the plasma concentration-time curve. Accordingly, the above trend would help explain why LCM/ND lipid nanoemulsion formulations (Figure 1) have been found capable of largely avoiding RES capture and display prolonged circulation in the bloodstream [2].

As specifically concerns neurodegenerative disease, vascular dementia (similarly to Alzheimer's disease) is a common cause of cognitive impairment - where there is an increased risk with aging, a rapid step-wise disease progression, and a high mortality rate [76]. The known cardiovascular risk factors driving vascular dementia include diabetes and hypertension. Accordingly, both clinical and experimental data indicate that associated cerebrovascular disease can lead to cerebral hypoperfusion, thereby altering brain metabolism and leading to cognitive impairment [76] (cf. [77]). Past studies (e.g., [78,79]) have already shown that low-grade inflammation and endothelial dysfunction contribute to reduced information processing speed and executive functioning in an older population. Biomedical application of colloidal drugnanocarriers, capable of crossing the BBB, can potentially be extended to the treatment of complex medical disorders like vascular dementia and (late-onset) Alzheimer's disease [78-82]. Recent published work has demonstrated that nanocomplexes can be readily transported into brain capillary endothelial cells (bovine and porcine) via SR-BI receptor-mediated endocytosis [7] (see also [83-85]). Accordingly, endothelial modulation and repair become feasible by pharmacological targeting[8-12,86-90] via SR-BI receptors (cf. $[13,90])$. The proposed multitasking combination therapeutic, described herein, appears likely to display greater efficacy at different stages of Alzheimer's disease (cf. [41]). Note also that this multitasking drugnanocarrier approach, i.e., multitasking drug-delivery vehicle [2] can also serve to reduce the size and/or extent of the "multidrug cocktail" [91] that the clinician would otherwise need to employ for adequate, or fully effective, treatment of the varied etiology of Alzheimer's-disease symptoms (i.e., the "multiple aging pathways" [91] or various pathogenic cascades involved). Moreover, the effects of the various cell types targeted via SR-BI [90] may be additive, multiplicative, or otherwise synergistic.

\section{Declarations}

Authors' contributions: Joseph S. D'Arrigo is the sole author of the article.
Acknowledgements: This research did not receive any specific grant from funding agencies in the public, commercial, or nonprofit sectors.

Conflict of interest: Author declares the following potential conflicts of interest:

1. J.S.D. is employed at Cav-Con Inc.

2. The actual "LCM/ND nanoemulsion (nanoparticle)" described in this review is not a finished/manufactured product, and is not on the retail market for sale.

\section{References}

1. D’Arrigo J S. Nanotargeting of Drug (s) for Delaying Dementia: Relevance of Covid-19 Impact on Dementia. American Journal of Alzheimer's Disease \& Other Dementias ${ }^{\circledR}, 2020,35: 1-12$,

2. D’Arrigo J S. Stable nanoemulsions: self-assembly in nature and nanomedicine. Amsterdam, The Netherlands:: Elsevier, 2011. 415 pp.

3. Tariq S, d'Esterre C D, Sajobi T T, et al. A longitudinal magnetic resonance imaging study of neurodegenerative and small vessel disease, and clinical cognitive trajectories in non demented patients with transient ischemic attack: the PREVENT study. BMC geriatrics, 2018, 18(1): 1-9.

4. Stefanova N A, Maksimova K Y, Rudnitskaya E A, et al. Association of cerebrovascular dysfunction with the development of Alzheimer's disease-like pathology in OXYS rats. BMC genomics, 2018, 19(3): 51-63.

5. Bennett R E, Robbins A B, Hu M, et al. Tau induces blood vessel abnormalities and angiogenesis-related gene expression in P301L transgenic mice and human Alzheimer's disease. Proceedings of the National Academy of Sciences, 2018, 115(6): E1289-E1298.

6. Duncombe J, Kitamura A, Hase Y, et al. Chronic cerebral hypoperfusion: a key mechanism leading to vascular cognitive impairment and dementia. Closing the translational gap between rodent models and human vascular cognitive impairment and dementia. Clinical Science, 2017, 131(19): 2451-2468.

7. Srimanee A, Regberg J, Hällbrink M, et al. Role of scavenger receptors in peptide-based delivery of plasmid DNA across a blood-brain barrier model. International journal of pharmaceutics, 2016, 500(1-2): 128-135.

8. Di Marco L Y, Venneri A, Farkas E, et al. Vascular dysfunction in the pathogenesis of Alzheimer's disease-a review of endothelium-mediated mechanisms and ensuing vicious circles. Neurobiology of disease, 2015, 82: 593606.

9. Carradori D, Gaudin A, Brambilla D, et al. Application of nanomedicine to the CNS diseases. International review of neurobiology, 2016, 130: 73-113.

10. Zenaro E, Piacentino G, Constantin G. The blood-brain barrier in Alzheimer's disease. Neurobiology of disease, 2017, 107: 41-56.

11. Qosa H, Mohamed L A, Al Rihani S B, et al. High-throughput screening for identification of blood-brain barrier 
integrity enhancers: a drug repurposing opportunity to rectify vascular amyloid toxicity. Journal of Alzheimer's Disease, 2016, 53(4): 1499-1516.

12. Koizumi K, Wang G, Park L. Endothelial dysfunction and amyloid- $\beta$-induced neurovascular alterations. Cellular and molecular neurobiology, 2016, 36(2): 155-165.

13. Goldwaser E L, Acharya N K, Sarkar A, et al. Breakdown of the cerebrovasculature and blood-brain barrier: A mechanistic link between diabetes mellitus and Alzheimer's disease. Journal of Alzheimer's Disease, 2016, 54(2): 445-456.

14. Mahringer A, Reichel V, Ott M, et al. Overcoming the Blood Brain Barrier-The Challenge of Brain Drug Targeting. Journal of Nanoneuroscience, 2012, 2(1): 5-19.

15. Fung K Y, Wang C, Nyegaard S, et al. SR-BI mediated transcytosis of HDL in brain microvascular endothelial cells is independent of caveolin, clathrin, and PDZK1. Frontiers in physiology, 2017, 8: 841.

16. Robert J, Button E B, Stukas S, et al. High-density lipoproteins suppress $A \beta$-induced PBMC adhesion to human endothelial cells in bioengineered vessels and in monoculture. Molecular neurodegeneration, 2017, 12(1): 1-19.

17. Robert J, Stukas S, Button E, et al. Reconstituted highdensity lipoproteins acutely reduce soluble brain $A \beta$ levels in symptomatic APP/PS1 mice. Biochimica Et Biophysica Acta (BBA)-Molecular Basis of Disease, 2016, 1862(5): 1027-1036.

18. Hottman D A, Chernick D, Cheng S, et al. HDL and cognition in neurodegenerative disorders. Neurobiology of disease, 2014, 72: 22-36.

19. Weekman E M, Sudduth T L, Caverly C N, et al. Reduced efficacy of anti-A $\beta$ immunotherapy in a mouse model of amyloid deposition and vascular cognitive impairment comorbidity. Journal of Neuroscience, 2016, 36(38): 9896-9907.

20. Nelson A R, Sweeney M D, Sagare A P, et al. Neurovascular dysfunction and neurodegeneration in dementia and Alzheimer's disease. Biochimica et Biophysica Acta (BBA)-Molecular Basis of Disease, 2016, 1862(5): 887900.

21. Kapasi A, Schneider J A. Vascular contributions to cognitive impairment, clinical Alzheimer's disease, and dementia in older persons. Biochimica et Biophysica Acta (BBA)-Molecular Basis of Disease, 2016, 1862(5): 878886.

22. McAleese K E, Alafuzoff I, Charidimou A, et al. Postmortem assessment in vascular dementia: advances and aspirations. BMC medicine, 2016, 14(1): 1-16.

23. Noh Y, Seo S W, Jeon S, et al. The role of cerebrovascular disease in amyloid deposition. Journal of Alzheimer's Disease, 2016, 54(3): 1015-1026.

24. Hishikawa N, Fukui Y, Sato K, et al. Cognitive and affective functions in Alzheimer's disease patients with metabolic syndrome. European journal of neurology, 2016, 23(2): 339-345.

25. Gutierrez J, Honig L, Elkind M S V, et al. Brain arterial aging and its relationship to Alzheimer dementia. Neurol- ogy, 2016, 86(16): 1507-1515.

26. Nagata K, Yamazaki T, Takano D, et al. Cerebral circulation in aging. Ageing Res. Rev. 2016, 30:49-60.

27. Calabrese V, Giordano J, Signorile A, et al. Major pathogenic mechanisms in vascular dementia: roles of cellular stress response and hormesis in neuroprotection. Journal of neuroscience research, 2016, 94(12): 1588-1603.

28. Toth P, Tarantini S, Csiszar A, et al. Functional vascular contributions to cognitive impairment and dementia: mechanisms and consequences of cerebral autoregulatory dysfunction, endothelial impairment, and neurovascular uncoupling in aging. American Journal of PhysiologyHeart and Circulatory Physiology, 2017, 312(1): H1-H20.

29. Devraj K, Poznanovic S, Spahn C, et al. BACE-1 is expressed in the blood-brain barrier endothelium and is upregulated in a murine model of Alzheimer's disease. Journal of Cerebral Blood Flow \& Metabolism, 2016, 36(7): 1281-1294.

30. Chao A C, Lee T C, Juo S H H, et al. Hyperglycemia increases the production of amyloid beta-peptide leading to decreased endothelial tight junction. CNS neuroscience \& therapeutics, 2016, 22(4): 291-297.

31. Khalil R B, Khoury E, Koussa S. Linking multiple pathogenic pathways in Alzheimer's disease. World journal of psychiatry, 2016, 6(2): 208.

32. Festoff B W, Sajja R K, van Dreden P, et al. HMGB1 and thrombin mediate the blood-brain barrier dysfunction acting as biomarkers of neuroinflammation and progression to neurodegeneration in Alzheimer's disease. Journal of neuroinflammation, 2016, 13(1): 1-12.

33. Gangoda S V S, Butlin M, Gupta V, et al. Pulsatile stretch alters expression and processing of amyloid precursor protein in human cerebral endothelial cells. Journal of Hypertension, 2016, 34:e24.

34. Roberts A M, Jagadapillai R, Vaishnav R A, et al. Increased pulmonary arteriolar tone associated with lung oxidative stress and nitric oxide in a mouse model of Alzheimer's disease. Physiological reports, 2016, 4(17): e12953.

35. Kyrtsos C R, Baras J S. Modeling the role of the glymphatic pathway and cerebral blood vessel properties in Alzheimer's disease pathogenesis. PloS one, 2015, 10(10): e0139574.

36. Kalaria R N, Akinyemi R, Ihara M. Stroke injury, cognitive impairment and vascular dementia. Biochimica et Biophysica Acta (BBA)-Molecular Basis of Disease, 2016, 1862(5): 915-925.

37. Khan A, Kalaria R N, Corbett A, et al. Update on vascular dementia. Journal of geriatric psychiatry and neurology, 2016, 29(5): 281-301.

38. Toda N, Okamura T. Cigarette smoking impairs nitric oxide-mediated cerebral blood flow increase: Implications for Alzheimer's disease. Journal of pharmacological sciences, 2016, 131(4): 223-232.

39. Uiterwijk R, Huijts M, Staals J, et al. Endothelial activation is associated with cognitive performance in patients with hypertension. American journal of hypertension, 2016, 29(4): 464-469.

40. Wang Y J. Lessons from immunotherapy for Alzheimer 
disease. Nature Reviews Neurology, 2014, 10(4): 188189.

41. Krstic D, Knuesel I. Deciphering the mechanism underlying late-onset Alzheimer disease. Nature Reviews Neurology, 2013, 9(1): 25.

42. D’Arrigo J S. Alzheimer's disease, brain injury, and CNS nanotherapy in humans: Sonoporation augmenting drug targeting. Medical Sciences, 2017, 5(4): 29.

43. Barbarese E, Ho S Y, D'Arrigo J S, et al. Internalization of microbubbles by tumor cells in vivo and in vitro. Journal of neuro-oncology, 1995, 26(1): 25-34.

44. Garg G, Saraf S, Saraf S. Cubosomes: an overview. Biological and Pharmaceutical Bulletin, 2007, 30(2): 350-353.

45. Pouton C W. Properties and uses of common formulation lipids, surfactants and cosurfactants//Proceedings of the AAPS Workshop, Effective Utilization of LipidBased Systems to Enhance the Delivery of Poorly Soluble Drugs: Physicochemical, Biopharmaceutical and Product Development Considerations, Bethesda, MD, USA. 2007: 5-6.

46. Kaasgaard T, Drummond C J. Ordered 2-D and 3-D nanostructured amphiphile self-assembly materials stable in excess solvent. Physical Chemistry Chemical Physics, 2006, 8(43): 4957-4975.

47. Small D M. The behavior of biological lipids. Pure and Applied Chemistry, 1981, 53(11): 2095-2103.

48. Seddon J M, Robins J, Gulik-Krzywicki T, et al. Inverse micellar phases of phospholipids and glycolipids. Invited Lecture. Physical Chemistry Chemical Physics, 2000, 2(20): 4485-4493.

49. Luzzati V, Vargas R, Mariani P, et al. Cubic phases of lipidcontaining systems: elements of a theory and biological connotations. Journal of molecular biology, 1993, 229(2): 540-551.

50. Luzzati V, Vargas R, Gulik A, et al. Lipid polymorphism: a correction. The structure of the cubic phase of extinction symbol Fd--consists of two types of disjointed reverse micelles embedded in a three-dimensional hydrocarbon matrix. Biochemistry, 1992, 31(1): 279-285.

51. D'arrigo J S. Surfactant mixtures, stable gas-in-liquid emulsions, and methods for the production of such emulsions from said mixtures: U.S. Patent 4,684,479. 1987-8-4.

52. D'arrigo J S. Method for the production of medical-grade lipid-coated microbubbles, paramagnetic labeling of such microbubbles and therapeutic uses of microbubbles: U.S. Patent 5,215,680993-6-1.

53. Nimmrich V, Eckert A. Calcium channel blockers and dementia. British journal of pharmacology, 2013, 169(6): 1203-1210.

54. Shirwany N A, Payette D, Xie J, et al. The amyloid beta ion channel hypothesis of Alzheimer's disease. Neuropsychiatric disease and treatment, 2007, 3(5): 597.

55. Di Scala C, Yahi N, Boutemeur S, et al. Common molecular mechanism of amyloid pore formation by Alzheimer's $\beta$-amyloid peptide and $\alpha$-synuclein. Scientific reports, 2016, 6(1): 1-10.

56. Demuro A, Smith M, Parker I. Single-channel $\mathrm{Ca}^{2+}$ imag- ing implicates $A \beta 1-42$ amyloid pores in Alzheimer's disease pathology. Journal of Cell Biology, 2011, 195(3): 515-524.

57. Serra-Batiste $M$, Ninot-Pedrosa $M$, Bayoumi $M$, et al. A $\beta 42$ assembles into specific $\beta$-barrel pore-forming oligomers in membrane-mimicking environments. Proceedings of the National Academy of Sciences, 2016, 113(39): 10866-10871.

58. Bode D C, Baker M D, Viles J H. Ion channel formation by amyloid- $\beta 42$ oligomers but not amyloid- $\beta 40$ in cellular membranes. Journal of Biological Chemistry, 2017, 292(4): 1404-1413.

59. Wang G H, Jiang Z L, Li Y C, et al. Free-radical scavenger edaravone treatment confers neuroprotection against traumatic brain injury in rats. Journal of neurotrauma, 2011, 28(10): 2123-2134.

60. Itoh T, Satou T, Nishida S, et al. Edaravone protects against apoptotic neuronal cell death and improves cerebral function after traumatic brain injury in rats. Neurochemical Research, 2010, 35(2): 348-355.

61. Jiao S S, Yao X Q, Liu Y H, et al. Edaravone alleviates Alzheimer's disease-type pathologies and cognitive deficits. Proceedings of the National Academy of Sciences, 2015, 112(16): 5225-5230.

62. Steinfeld B, Scott J, Vilander G, et al. The role of lean process improvement in implementation of evidence-based practices in behavioral health care. The Journal of Behavioral Health Services \& Research, 2015, 42(4): 504518.

63. Daulatzai M A. Cerebral hypoperfusion and glucose hypometabolism: key pathophysiological modulators promote neurodegeneration, cognitive impairment, and Alzheimer's disease. Journal of neuroscience research, 2017, 95(4): 943-972.

64. D'Arrigo J S. Biomaterial to improve drug delivery in Alzheimer's disease: Linking major pathogenic pathways. OBM Geriatrics, 2020, 4(1):10.

65. Gambini J. Oxidative stress and inflammation: from mechanisms to therapeutic approaches. Biomedicines, 2020.

66. D’Arrigo J S. Biomimetic nanocarrier targeting drug (s) to upstream-receptor mechanisms in dementia: Focusing on linking pathogenic cascades. Biomimetics, 2020, 5(1): 11.

67. Tangestani Fard M, Stough C. A review and hypothesized model of the mechanisms that underpin the relationship between inflammation and cognition in the elderly. Frontiers in aging neuroscience, 2019, 11: 56.

68. Khalil A, Berrougui H, Pawelec G, et al. Impairment of the ABCA1 and SR-BI-mediated cholesterol efflux pathways and HDL anti-inflammatory activity in Alzheimer's disease. Mechanisms of ageing and development, 2012, 133(1): 20-29.

69. Thanopoulou K, Fragkouli A, Stylianopoulou F, et al. Scavenger receptor class B type I (SR-BI) regulates perivascular macrophages and modifies amyloid pathology in an Alzheimer mouse model. Proceedings of the National Academy of Sciences, 2010, 107(48): 20816- 
20821.

70. D’Arrigo J S. Delaying dementia: Targeted brain delivery using lipid cubic phases. OBM Neurobiol, 2019, 3.

71. Broderick T L, Rasool S, Li R, et al. Neuroprotective Effects of Chronic Resveratrol Treatment and Exercise Training in the 3xTg-AD Mouse Model of Alzheimer's Disease. International journal of molecular sciences, 2020, 21(19): 7337.

72. Ma X R, Sun Z K, Han X, et al. Neuroprotective effect of resveratrol via activation of Sirt1 signaling in a rat model of combined diabetes and Alzheimer's disease. Frontiers in neuroscience, 2020, 13: 1400.

73. Santos A C, Sequeira J A D, Pereira I, et al. Sonicationassisted Layer-by-Layer self-assembly nanoparticles for resveratrol delivery. Materials Science and Engineering: C, 2019, 105: 110022.

74. Xiao K, Li Y, Luo J, et al. The effect of surface charge on in vivo biodistribution of PEG-oligocholic acid based micellar nanoparticles. Biomaterials, 2011, 32(13): 34353446.

75. Maeda H, Seymour L W, Miyamoto Y. Conjugates of anticancer agents and polymers: advantages of macromolecular therapeutics in vivo. Bioconjugate chemistry, 1992, 3(5): 351-362.

76. Baik S H, Selvaraji S, Fann D Y, et al. Hippocampal Transcriptome Profiling Reveals Common Disease Pathways in Chronic Hypoperfusion and Ageing. bioRxiv, 2020.

77. Salminen A. The suppressive mechanisms are examined in detail in association with AD pathogenesis Hypoperfusion is a potential inducer of immunosuppressive network in Alzheimer's disease. Neurochemistry international, 2020: 104919.

78. Heringa S M, van den Berg E, Reijmer Y D, et al. Markers of low-grade inflammation and endothelial dysfunction are related to reduced information processing speed and executive functioning in an older population-the Hoorn study. Psychoneuroendocrinology, 2014, 40: 108-118.

79. D'Arrigo J S. Nanotargeting dementia etiology: Aiming drug nanocarriers toward receptors for vascular endothelium, serum amyloid A, inflammasomes, and oxidative stress. Nano Progress, 2020, 2(3):25-30.

80. Lénárt N, Brough D, Dénes Á. Inflammasomes link vascular disease with neuroinflammation and brain disorders. Journal of Cerebral Blood Flow \& Metabolism, 2016,
36(10): 1668-1685.

81. Sierksma A, Lu A, Mancuso R, et al. Novel Alzheimer risk genes determine the microglia response to amyloid- $\beta$ but not to TAU pathology. EMBO molecular medicine, 2020, 12(3): e10606.

82. Matsumoto J, Dohgu S, Takata F, et al. Serum amyloid Ainduced blood-brain barrier dysfunction associated with decreased claudin-5 expression in rat brain endothelial cells and its inhibition by high-density lipoprotein in vitro. Neuroscience Letters, 2020, 738: 135352.

83. Lajoie J M, Shusta E V. Targeting receptor-mediated transport for delivery of biologics across the blood-brain barrier. Annual review of pharmacology and toxicology, 2015, 55: 613-631.

84. Almer G, Mangge H, Zimmer A, et al. Lipoprotein-related and apolipoprotein-mediated delivery systems for drug targeting and imaging. Current medicinal chemistry, 2015, 22(31): 3631-3651.

85. Preston J E, Abbott N J, Begley D J. Transcytosis of macromolecules at the blood-brain barrier. Advances in pharmacology, 2014, 71: 147-163.

86. Salmina A B, Inzhutova A I, Malinovskaya N A, et al. Endothelial dysfunction and repair in Alzheimer-type neurodegeneration: neuronal and glial control. Journal of Alzheimer's Disease, 2010, 22(1): 17-36.

87. Tong X K, Hamel E. Simvastatin restored vascular reactivity, endothelial function and reduced string vessel pathology in a mouse model of cerebrovascular disease. Journal of Cerebral Blood Flow \& Metabolism, 2015, 35(3): 512-520.

88. Koster K P, Thomas R, Morris A W J, et al. Epidermal growth factor prevents oligomeric amyloid- $\beta$ induced angiogenesis deficits in vitro. Journal of Cerebral Blood Flow \& Metabolism, 2016, 36(11): 1865-1871.

89. Hostenbach S, D'haeseleer M, Kooijman R, et al. The pathophysiological role of astrocytic endothelin-1. Progress in neurobiology, 2016, 144: 88-102.

90. Tran-Dinh A, Levoye A, Couret D, et al. High-Density Lipoprotein Therapy in Stroke: Evaluation of Endothelial SR-BI-Dependent Neuroprotective Effects. International Journal of Molecular Sciences, 2021, 22(1): 106.

91. Ladiges W C. Time for an Alzheimer's disease cocktail. Aging Pathobiology and Therapeutics, 2020, 2(1): 14-15.

Cite this article as: Joseph S D. Drug nanotargeting for treatment of neurodegeneration and aging[J]. Aging Pathobiology and Therapeutics, 2021, 3(2): 20-27. 